\title{
EL REQUISITO OBLIGATORIO DEL SERVICIO SOCIAL EN SALUD DEL PERÚ: DISCRIMINATORIO E INCONSTITUCIONAL
}

\author{
Percy Mayta-Tristán ${ }^{1, a}$, Julio A. Poterico 2,b, Edén Galán-Rodas,a, Daniel Raa-Ortiz ${ }^{4,5, c}$
}

\begin{abstract}
RESUMEN
El servicio rural y urbano marginal en salud (SERUMS) es una actividad que realizan solo los profesionales de la salud al Estado peruano, ya que constituye un requisito obligatorio para optar por la segunda especialidad o para trabajar en un centro de salud público, y obtener becas del gobierno para futura capacitación. Los escasos cambios legales en el reglamento de este programa social y el enfoque de "servicio" restringido a los profesionales de salud conllevan a que esta política sea discriminatoria e inconstitucional por atentar contra el derecho a la educación y al trabajo. No hay evidencia científica que sustente la utilidad y efectividad de este programa, tanto en la calidad de servicio y mejora de indicadores sanitarios, como en la adecuada distribución y retención de profesionales de salud. Sugerimos abolir el requisito de obligatoriedad y replantear una estrategia política que ayude a atraer y retener a los profesionales de la salud en zonas vulnerables del Perú.
\end{abstract}

Palabras clave: Recursos humanos; Distribución de médicos; Trabajadores rurales; Salud pública; Políticas; Perú (fuente: DeCS BIREME).

\section{MANDATORY REQUIREMENT OF SOCIAL HEALTH SERVICE IN PERU: DISCRIMINATORY AND UNCONSTITUTIONAL}

\begin{abstract}
RESUMEN
The rural and urban-edge health service (SERUMS) is an activity that only health professionals perform for the Peruvian government, as it is a mandatory requirement to qualify for a second specialty or to work in public hospitals and public health care facilities, and obtain government scholarships for future training. The few legal changes in the rules of this social program and the focus of "service" restricted to health professionals lead to a perception of this policy as discriminatory and unconstitutional because it violates the right to education and work. There is no scientific evidence that supports the usefulness and effectiveness of this program in terms of quality of service and health indicator improvement, as well as in adequate distribution and retention of health professionals. We suggest to abolish the compulsory requirement and to reformulate a political strategy to help attract and retain health professionals in vulnerable areas of Peru.
\end{abstract}

Key words: Human resources; Physician distribution; Rural workers; Public health; Policy; Peru (source: MeSH NLM)

\section{INTRODUCCIÓN}

Uno de los retos más importantes para los sistemas de salud en el mundo es lograr una adecuada distribución de los recursos humanos en salud (RHUS) (1). Sin embargo, pocos sistemas lo logran y como consecuencia se enfrenta una distribución inequitativa de los RHUS, perjudicando a las zonas rurales y urbanas marginales ${ }^{(2)}$. Por esta razón, los gobiernos han tratado de generar o adaptar estrategias para atraer y retener al personal de salud ${ }^{(3-5)}$.

En la mayoría de países de Latinoamérica se crearon los servicios sociales en medicina como una forma

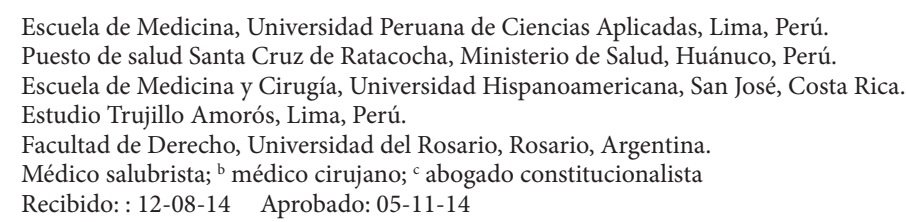


de respuesta ante la escasez de RHUS en las zonas vulnerables ${ }^{(6)}$. El primero se generó en 1936 en la Universidad Nacional Autónoma de México, posterior a la Revolución Mexicana, donde se planteó como un acto de reciprocidad que todos los egresados debían pasar una temporada en los servicios rurales para "retribuir a la sociedad la deuda que contraían al haberlos educado" (7,8). Esta iniciativa se adoptó luego como política en México en un escenario donde solo había formación médica por universidades públicas (6). Otros países de Latinoamérica implementaron políticas similares, pero con diferentes perspectivas, siendo un pilar para su implementación, la necesidad de cumplir este programa (obligatoriedad) por los graduandos/profesionales de salud para poder obtener licencia para ejercer su actividad en el territorio nacional, trabajar para alguna institución del Estado, realizar un programa de posgrado, obtener becas financiadas por el gobierno respectivo, entre otros ${ }^{(6)}$.

En el Perú se implementó en 1972 el Servicio Civil de Graduandos (SECIGRA) en Ciencias de la Salud (SECIGRA-Salud) como piloto del servicio social de graduandos ${ }^{(9)}$, imitando la iniciativa pionera mexicana. Este programa cambiaría de denominación a Servicio Rural y Urbano Marginal De Salud (SERUMS) en $1981^{(10)}$; con una reglamentación establecida en el año $1997^{\left({ }^{(11)} .\right.}$ La ley establece que el SERUMS representa un requisito indispensable para ocupar cargos en entidades públicas, ingresar a los programas de segunda especialización profesional y recibir del Estado beca u otra ayuda equivalente para estudios o perfeccionamiento (10). El reglamento incluye a los siguientes profesionales: médicos, odontólogos, enfermeros, obstetras, químicofarmacéuticos, nutricionistas, tecnólogos médicos, asistentes sociales, biólogos, psicólogos, veterinarios e ingenieros sanitarios ${ }^{(11)}$.

En el Perú, se lograron establecer otros SECIGRA para Derecho ${ }^{\left({ }^{12}\right)}$ e Ingenierías ${ }^{(13)}$. Sin embargo, se derogaron o dejaron de ser obligatorios ${ }^{(14)}$, y en la actualidad tienen un carácter más parecido al internado de las ciencias de la salud; es decir, son períodos de 6 a 12 meses en instituciones públicas en su misma ciudad y continuando con la supervisión de la universidad a la que pertenece el estudiante ${ }^{(14)}$.

En Colombia (15) y México (7), con un servicio social en medicina similar al Perú, se está cuestionando la utilidad de esta política como herramienta de distribución de RHUS y mejora de la atención sanitaria. En este artículo plantearemos una mirada crítica al SERUMS en el Perú, en particular al requisito obligatorio, que consideramos que discriminatorio e inconstitucional.

\section{EVIDENCIAS DISPONIBLES SOBRE EL SERUMS}

EI SERUMS tiene más de cuatro décadas de funcionamiento desde su implementación como SECIGRA. Al realizar búsquedas en SciELO, PUBMED, SCOPUS, Scholar Google, Biblioteca Virtual en Salud y en páginas del Ministerio de Salud del Perú, no se encuentra evidencia alguna que mida cuál sería el efecto de esta intervención, y cuán favorable ha sido para mejorar la salud de las poblaciones vulnerables. Situación similar a lo que referencian otros investigadores del tema en la cuna de este programa en el sector salud: México ${ }^{(7)}$.

En una revisión realizada por la Organización Panamericana de la Salud (OPS) sobre estos programas sociales de medicina ${ }^{(6)}$, se han identificado dificultades superables que, a pesar de los años, se mantienen desde su establecimiento (Tabla 1). Por otra parte, en el Perú se encuentran reportes que confirman y agregan problemas a la lista mencionada.

Entre las falencias y riesgos reportados en el SERUMS peruano se encuentran: un deficiente aseguramiento en salud así como contra accidentes y muertes para los que realizan este servicio ${ }^{(16)}$, serumistas con una salud mental alterada ${ }^{(17)}$ y una de las tasas de mortalidad de médicos recién egresados más grande del mundo,

Tabla 1. Áreas críticas del servicio social de salud en Latinoamérica ${ }^{(6)}$

\begin{tabular}{l}
\hline Áreas críticas \\
\hline Falta de una política tangible y dinámica de recursos \\
humanos en salud \\
Obligatoriedad del servicio \\
Falta de compromiso e identificación \\
Desarticulación de docencia, servicio e investigación. \\
Tutoría, supervisión y coordinación deficiente o inexistente \\
Falta de actualización, ausencia o inconsistencia entre \\
normas propuestas para este programa \\
Peligro real de desaparición \\
Inadecuadas y hasta riesgosas condiciones laborales en las \\
zonas de servicio \\
Falta de presupuesto destinado a este tipo de servicio para \\
su mejora continua y mayor efectividad \\
Participación inefectiva de las comunidades \\
Dificultad para su desarrollo en la organización social \\
existente: subsistemas desarticulados, desorganizados y \\
burocracia ineficiente \\
Falta de coordinación entre los colegios profesionales, las \\
universidades y los ministerios \\
Discrepancia entre misión y objetivos entre la academia y el \\
Estado. Falta de una visión clara
\end{tabular}


principalmente por accidentes de carretera al transportar pacientes en emergencia ${ }^{(18,19)}$.

Otra debilidad existente representa la discordancia entre lo aprendido en las universidades y lo necesario para trabajar en el primer nivel de atención, particularmente en el manejo de los programas que se tiene a cargo, así como en los aspectos de gestión y administración en salud ${ }^{(20,21)}$. Además, al ir a zonas rurales se tienen problemas con el trato a la población por una deficiencia del manejo multicultural y participativo, así como las dificultades del ambiente laboral en ciertos casos ${ }^{(22)}$. Los problemas descritos -y otros sin describir pero vivenciados-no son exclusivos de los profesionales de la salud peruanos, también han sido reportados en Colombia ${ }^{(15,23)}$ y México ${ }^{(6,24-26)}$. Incluso en Sudáfrica se refieren al servicio social de medicina como un periodo de "esclavitud" necesario para seguir con el desarrollo profesional (27). Esto puede explicar la falta de atracción y la incapacidad en retener a los serumistas para trabajar en el futuro en las zonas vulnerables ${ }^{(28)}$; con una "bola de nieve" de comentarios que influye en las decisiones de los futuros serumistas y profesionales que hayan culminado este programa ${ }^{(29)}$.

La duración y el momento de inicio del SERUMS puede ser también un problema tanto para la población como para el serumista. Hay dos momentos de ingreso al SERUMS por año, en mayo -con mayor cantidad de plazas- y octubre, siendo la duración del programa de doce meses. Hay que tener en cuenta que en un buen porcentaje de casos el médico serumista es el jefe del centro de salud, por lo que tiene que ejecutar un presupuesto y elaborar un plan de gestión anual. Cuando se ingresa, los serumistas ya tienen un presupuesto y plan aprobado por otro serumista del año anterior, y luego generan uno para el siguiente año que lo ejecutará otro, por lo que su capacidad de gestión y de evaluación de resultados es bastante limitada, dado el corto periodo de aprendizaje y aplicación de lo aprendido en el servicio.

Por otro lado, la necesidad de participar en el examen de residentado médico, conlleva a que muchos de los serumistas médicos dediquen un gran porcentaje de su tiempo en la actividad de preparación para esta prueba transversal de conocimientos que suele realizarse al mes-o a los 8 meses para la segunda convocatoria de SERUMS — de terminado este programa de servicio en salud.

Por lo expuesto, el programa SERUMS pareciera haber fallado en cumplir la premisa de llevar los servicios de salud de calidad y calidez a las poblaciones vulnerables del país, y se sugeriría un cambio urgente de este servicio social de los profesionales de salud.

\section{SERUMS COMO REQUISITO DISCRIMINATORIO E INCONSTITUCIONAL}

Como se mencionó, el SERUMS solo involucra a algunas profesiones del sector salud. En contraste, el SECIGRA en Derecho dejó de ser obligatorio en el año $2002{ }^{(14)}$ y ninguna otra profesión en el Perú necesita hacer un año de trabajo en el Estado. Con lo expuesto, planteamos la necesidad de evaluar si las normas jurídicas que regulan el SERUMS son inconstitucionales $y$, en este sentido, cuáles son las soluciones legales posibles que se pueden adoptar en la sociedad o en aquellos profesionales de la salud involucrados.

La Constitución Política del Perú señala en el numeral 15 de su artículo $2 .^{\circ}$ que toda persona tiene derecho a la libertad de trabajo ${ }^{(30)}$, que según el Tribunal Constitucional peruano es "El atributo para elegir a voluntad la actividad ocupacional o profesional que cada persona desee o prefiera desempeñar, disfrutando de su rendimiento económico y satisfacción espiritual; así como cambiarla o de cesar de ella" ${ }^{(31)}$. Es decir, la libertad de trabajo permite la decisión de la persona en qué, dónde, cómo y hasta cuándo trabajar. Nadie la puede obligar a trabajar, ni siquiera el Estado, quien debe garantizar esta libertad de decisión. De allí que podemos concluir que el SERUMS, tal como está configurado, atenta contra esa capacidad de elegir del profesional de salud respecto a la forma, modo y términos en el cual decida desarrollar su trabajo en su campo respectivo.

Una posición contraria sostendría que la libertad de trabajo debe ser restringida, debido a que el SERUMS tutela el derecho a la salud de los ciudadanos en zonas vulnerables, siendo posible que el Estado pueda obligar al profesional de salud a prestar dicho servicio. Esta posición plantearía que frente a un derecho social como la salud debe limitarse el derecho personal del profesional. Cuando hay conflicto de derechos fundamentales, se aplica el test de proporcionalidad, que fue utilizado por el Tribunal Constitucional en el caso del Servicio Militar Obligatorio ${ }^{(32)}$, otra forma de prestación de servicios impositiva a favor del Estado, similar al SERUMS. Bajo este análisis el SERUMS no cumpliría con: (a) el subprincipio de necesidad, que plantea que para que exista una injerencia en un derecho fundamental, no debe existir otro medio alternativo que permita el mismo objetivo, pero que sea más benigno que el derecho afectado; y, (b) el subprincipio de proporcionalidad en sentido estricto, que supone en sopesar los derechos constitucionales en conflicto y determinar cuál de ellos tiene mayor peso sobre el otro ${ }^{(32)}$. Debido a que existen otros mecanismos para distribuir los RHUS a las zonas 
vulnerables, planteamos como injustificado vulnerar la libertad de decisión del profesional de la salud con relación a su trabajo; y también para condicionar al SERUMS como un requisito para trabajar en el Estado o acceder a la formación de segunda especialización.

Otras opiniones podrían sostener que en realidad el fundamento del SERUMS es la reciprocidad (8), es decir, devolver a la sociedad por lo que se hizo para la formación de los RHUS. Como se refería anteriormente, estos programas de servicio social se dieron en un contexto en el cual solo existía la universidad pública. Por tanto, ¿por qué un estudiante de una universidad privada tendría que hacer SERUMS? Si concluimos, llegando al absurdo de dicho argumento, que solo los estudiantes de universidades públicas deben realizar SERUMS, entonces ¿por qué únicamente los estudiantes de salud lo deben realizar y se excluye a otras profesiones? De esta manera, estamos ante un trato discriminatorio que atenta contra el derecho del principio de igualdad consagrado en el numeral 2 del artículo 2 de la Constitución Política del Perú, que exige al Estado abstenerse de la producción legal de diferencias arbitrarias o caprichosas; tal como lo ha sentenciado el Tribunal Constitucional previamente al definir "igualdad formal" (33).

Si bien no es obligatorio hacer SERUMS para ejercer las profesiones de salud, es un requisito para acceder a programas de especialización o para trabajar en el Estado ${ }^{(10,11)}$. Esto también es inconstitucional por afectar al derecho a la educación, debido a que el Estado debe velar por el acceso a una educación adecuada para todos los ciudadanos conforme al artículo 16 de la Constitución peruana ${ }^{(30)}$; sin tratos diferenciados frente a otros estudiantes, sean de universidades privadas 0 de otras carreras profesionales. Por tanto, consideramos que el SERUMS no debería ser un requisito obligatorio, sino facultativo, como sucede con el SECIGRADerecho (14). Esto únicamente debería proceder a través de una modificación normativa por una iniciativa legislativa ante el Congreso de la República (artículo 107 de la Constitución), teniendo en consideración los argumentos anteriores, debido que actualmente no se puede solicitar la inconstitucionalidad de la norma ante el Tribunal Constitucional al haber prescrito el plazo de seis años establecido en las leyes peruanas ${ }^{(34)}$.

Ello no impide que una persona que se considere afectada en sus derechos constitucionales por la aplicación del SERUMS solicite vía proceso constitucional de Amparo, conforme al numeral 2 del artículo 200 de la Constitución, la inaplicación judicial del mismo. Pero los efectos legales se aplicarían únicamente para el ciudadano, salvo que el proceso judicial sea de conocimiento del Tribunal Constitucional, quien en ejercicio de sus competencias emita un precedente vinculante que permita únicamente en casos futuros la inaplicación por inconstitucionalidad del SERUMS. Esta alternativa también podría ser acogida por alguno de los colegios profesionales que se sientan afectados.

\section{ALTERNATIVAS PARA DISTRIBUIR Y RETENER RECURSOS HUMANOS EN SALUD EN ZONAS VUNERABLES MEDIANTE EL SERVICIO SOCIAL EN SALUD}

Mientras que varios países latinoamericanos apostaron por los servicios sociales obligatorios, Chile en 1955 crea el programa "Médicos Generales de Zona" (MGZ), partiendo de la evaluación de los servicios sociales que tenían poca retención de los médicos en las áreas rurales luego de su año obligatorio ${ }^{(35)}$. En otras palabras, desde hace más de medio siglo el gobierno chileno adoptó medidas de incentivo, atracción y retención efectivas para la distribución de los RHUS, sobre todo de médicos.

El programa MGZ tiene como objetivo atraer y retener a médicos al trabajo rural en centros de salud del primer nivel de atención por un periodo mínimo de tres años (y un máximo de seis), teniendo como mayor incentivo el realizar una residencia pagada en un hospital universitario, ya que en Chile los médicos residentes que ingresan bajo la modalidad de examen no reciben honorarios. Además, tienen salarios atractivos y otros beneficios que se dan proporcionalmente al grado de aislamiento y su responsabilidad clínico-administrativa. Sus contratos no obligan la permanencia con un tiempo establecido, pudiendo postular únicamente a la residencia, bajo esta modalidad, si cumplen con el periodo mínimo de tres años ${ }^{(36)}$. En un análisis realizado sobre el programa para el periodo 2004-2009 se encontró que la cantidad de postulantes excedió las plazas ofertadas y todas fueron cubiertas: $90 \%$ de los MGZ refieren una alta satisfacción, y la permanencia hasta los 6 años llega hasta un $58 \%$ de casos ${ }^{(36)}$.

En Perú se han planteado varios proyectos de Ley para reemplazar el SERUMS, entre ellos el Proyecto Servicio de Medicina y Salud Comunitaria (SERMEC) presentado en el $2005^{(37)}$, el cual plantea un programa de profesionales de la salud para zonas rurales y urbanas marginales, que duran más de un año y exime la obligatoriedad del SERUMS para las actividades restrictivas actuales en el marco legal de este programa en Perú. 
Para incrementar el número de profesionales de la salud en áreas vulnerables hay varios factores involucrados, que se pueden dividir en condiciones previas del sujeto que favorecen la decisión, los mecanismos de reclutamiento y de retención ${ }^{(3-5,38)}$. Los primeros involucran el reclutamiento de personas procedentes de zonas rurales para estudiar en profesiones de salud, el incluir actividades en zonas rurales en las mallas curriculares universitarias o como actividades extraacadémicas, conocer las intenciones de trabajo y permanencia en zonas rurales, entre otros ${ }^{(4)}$. En este aspecto se podría trabajar una política que integra la formación de profesionales en salud con orientación de primer nivel de atención, al tener mayor interacción entre el Estado, la universidad y los colegios profesionales.

El reclutamiento y retención dependen directamente de las políticas que implementen cada Estado, como principal empleador en el sector salud. El modelo del SERUMS como principal política peruana para la distribución de RHUS asegura el reclutamiento mas no la retención, con una gran movilidad y recambio del personal de salud en las zonas más vulnerables del Perú; mermando la sostenibilidad de los programas y estrategias sanitarias. Basado en lo discutido, creemos que el SERUMS debe transformarse en una verdadera política de distribución de RHUS que considere algo más que el reclutamiento y cobertura; incluyendo entre otros aspectos, los siguientes puntos:

a) Debe ser un programa voluntario. No ser requisito para optar una segunda especialización, ni para trabajar en el Estado o recibir alguna beca o subvención. En cambio, sí puede ser considerado como una bonificación para los concursos públicos.

b) Los establecimientos de salud que reciban profesionales deben contar con condiciones mínimas de infraestructura, seguridad, salubridad y acceso; procurando contar con el equipo básico de atención primaria de salud según el nivel del establecimiento

c) Los programas de incentivos económicos deben ser proporcionales al aislamiento y responsabilidad del profesional.

d) Los contratos no deben tener un periodo mínimo de estancia, pero los incentivos académicos se deberían dar a partir de un mínimo de 3 años de estancia.

e) El acceso a la segunda especialización (residencia en medicina o similar para otras profesiones) debe ser en un concurso separado para aquellos que accedan por esta vía, similar a lo que actualmente se viene dando en las plazas de destaque. Con un puntaje diferenciado según las actividades que haya desarrollado, incluyendo: publicaciones científicas, capacitaciones, reconocimientos, proyectos sociales a cargo, entre otros.

f) Las instituciones prestadoras de servicios del sistema de salud articuladas con la academia deben implementar programas de capacitación y asistencia permanente a los profesionales, mejorando sus competencias en aspectos de gestión, administración y ayuda diagnóstica, entre otros pertinentes.

g) Se debería evaluar la posibilidad de que una vez lograda una temática formativa concertada con los gobiernos regionales, las universidades y los colegios profesionales, los serumistas puedan brindar sus servicios además del ámbito asistencial en los campos de la administración y gestión en salud; así como en temas de salud pública e investigación en tópicos de la atención primaria, entre otros necesarios.

\section{CONCLUSIONES}

EI SERUMS representa un servicio social que realizan los profesionales de la salud excluyendo a otras carreras; limitando el abordaje en salud pública multidisciplinario, con un enfoque meramente paternalista que intenta tener presencia aunque poco interés en generar impacto significativo en las comunidades más vulnerables del Perú. Además, la condición de obligatoriedad restringida a las carreras de salud es discriminatoria e inconstitucional por atentar contra el derecho a la educación y al trabajo.

No hay evidencia científica que sustente la utilidad y efectividad de este programa tanto en la calidad de servicio y mejora de indicadores sanitarios; como en la adecuada distribución y retención de profesionales de salud en las zonas más vulnerables. Si bien es cierto que el Estado debe velar por la salud de su población, debe tenerse en cuenta que la estrategia del SERUMS merece mayor atención, tanto por su incertidumbre como intervención, como por su efecto en los derechos fundamentales de los profesionales de la salud. Urgen cambios normativos para lograr atraer, incentivar y retener a los profesionales de la salud en las zonas rurales y urbanas marginales del Perú (39,40). Se podría comenzar por convertir el servicio en facultativo, y luego planificar, coordinar y articular las actividades prioritarias con los gobiernos regionales, las universidades y los colegios profesionales. Concomitantemente, se debería brindar las facilidades y motivaciones para el desenvolvimiento máximo durante el servicio social en salud, continuándose con estas iniciativas a lo largo del desempeño del profesional sanitario una vez culminado este programa. 
Contribuciones de autoría: PMT y JP diseñaron la estructura, hicieron la búsqueda de información y redactaron la versión preliminar, DRO hizo el análisis legal, EGR revisó críticamente el contenido, todos los autores aprobaron la versión final a publicar.
Conflictos de interés: JAP actualmente cursa el SERUMS, EGR y PMT realizaron hace varios años el SERUMS y DRO no realizó el servicio social porque en Derecho el SECIGRA no es obligatorio.

Financiamiento: autofinanciado.

\section{REFERENCIAS BIBLIOGRÁFICAS}

1. World Health Organization. Working together for health. The World Health Report 2006. Geneva: WHO; 2006.

2. Hongoro C, McPake B. How to bridge the gap in human resources for health. Lancet. 2004 Oct 1622;364(9443):1451-6.

3. Wilson NW, Couper ID, De Vries E, Reid S, Fish T, Marais BJ. A critical review of interventions to redress the inequitable distribution of healthcare professional to rural and remotes areas. Rural Remote Health. 2009 AprJun;9(2):1060.

4. Dolea C, Stormont L, Braichet JM. Evaluated strategies to increase attraction and retention of health workers in remote and rural areas. Bull World Health Organ. 2010 May;88(5):379-85. doi: 10.2471/ BLT.09.070607.

5. Frehywot S, Mullan F, Payne PW, Ross H. Compulsory service programmes for recruiting health workers in remote and rural areas: do they work? Bull World Health Organ. 2010;88(5):36470.

6. Organización Panamericana de la Salud. El Servicio Social de Medicina en Latinoamérica: situación actual y perspectivas. Serie Desarrollo de Recursos Humanos No 18. Washington DC: OPS; 1998.

7. Nigenda G. Servicio social en medicina en México. Una reforma urgente y posible. Salud Publica Mex. 2013 SepOct;55(5):519-27.

8. Mungaray A, Ocegueda JM, Sanchez MD. Retos y perspectivas de la reciprocidad universitaria a través del servicio social en México. México DF: Universidad Autónoma de Baja California; 2002.

9. Perú, Congreso de la República. Decreto Ley 19646. Servicio Civil de Graduandos en Ciencias de la Salud Humana (SECIGRA-Salud). Lima: Congreso de la República; 1972.

10. Perú, Congreso de la República.
Decreto Ley 23330 Ley del Servicio Rural y Urbano Marginal de Salud - SERUMS. Lima: Congreso de la República; 1981.

11. Perú, Ministerio de Salud. Decreto Supremo 005-97-SA. Reglamento de la Ley 23330, Ley del Servicio Rural y Urbano Marginal de Salud - SERUMS. Lima: MINSA; 1997.

12. Perú, Congreso de la República. Decreto Ley 21824 Establecen Servicio Civil de Graduandos en Sector Vivienda (SECIGRA-Vivienda). Lima: Congreso de la República; 1977.

13. Perú, Congreso de la República. Decreto Ley 25647 Servicio Civil de Graduandos en Derecho (SECIGRADerecho). Lima: Congreso de la República; 1992.

14. Perú, Congreso de la República. Decreto Ley 27687 Ley que otorga carácter facultativo al SECIGRADerecho. Lima: Congreso de la República; 2002.

15. Moya-Peñafiel MJ. Servicio social obligatorio en Colombia: incertidumbre de los recién graduados en medicina. Rev Med Risaralda. 2014;20(2):114-120.

16. Mejia CR, Quiñones-Laveriano DM, Espinoza KG, Quezada-Osoria C. Deficiente cobertura de aseguramiento a médicos durante el servicio rural y urbano-marginal en Perú. Rev Peru Med Exp Salud Publica, 2013;30(2):220-3.

17. Galán-Rodas E, Gálvez-Buccollini JA, Vega-Galdós F. Salud mental en médicos que realizan el servicio rural, urbano-marginal en salud en el Perú: Un estudio de línea base. Rev Peru Med Exp Salud Publica. 2011;28(2):277-81.

18. Galán-Rodas E, Díaz-Vélez C, Villena J, Maguiña C. Mortalidad de médicos que realizan el servicio rural (SERUMS) en Perú, 2006-2009. Rev Peru Med Exp Salud Publica. 2010;27(3):483-4.

19. Wong PA. Los nuevos mártires de la medicina en el Perú. An Fac Med. 2009;70(2):151-2.
20. Motta F, Frisancho A. La experiencia serums y la formación profesional. Rev Peru Epidemiol. 1992;5(2):24-8.

21. Valencia T, Leon B, Lezameta U, Vidal M. Principales dificultades del profesional de salud encontrados durante el Serums en el departamento de Ancash, zona sierra. 2008. Rev Aporte Santiaguino. 2011;4(1):11822.

22. Reyes E. "Allá es clínicamente así: saber llegar" de la formación a la práctica profesional médica. El Servicio Rural Urbano Marginal en Salud (SERUMS). Apuntes. 2011;38(69):55-80.

23. Arango Y, Pedroza A, Balmas G, Erazo L, Lopez R. Atención primaria y educación médica: un aprendizaje vivencial a través ruralito. Colombia. Educ Med Salud. 1987;21(1):70-81.

24. Lopez J, Gonzalez de Cossio MG, Velasco M. Servicio social de medicina en Mexico. Factibilidad del cumplimiento académico en el área rural. Rev Fac Med UNAM. 2004; 47(5):181-6.

25. Hamui-Sutton A. Condiciones sociales en que los pasantes de la Facultad de Medicina (FM) de la Universidad Nacional Autónoma de Mexico (UNAM) realizan el Servicio Social en áreas rurales. Gac Med Mex. 2012 MayJun;148(3):277-80.

26. Ramirez OF, Hamui A, Reynaga J, Varela M, Ponce de León ME, Diaz A, et al. Condiciones de trabajo de los médicos pasantes mexicanos durante el servicio social. Perfiles Educ. 2012;34(138):92-107.

27. Erasmus N. Slaves of the state - medical internship and community service in South Africa. SAMJ. 2012;102(8):6558.

28. Huicho L, Canseco FD, Lema C, Miranda JJ, Lescano AG. Incentivos para atraer y retener personal de salud de zonas rurales del Perú: un estudio cualitativo. Cad Saude Publica. 2012 Apr;28(4):729-39. 
29. Mayta-Tristan P, Mejia CR, RiegaLopez P, Rojas-Mezarina L, Posso M, Mezones-Holguin E. Proyección de trabajo en el interior del país y factores asociados en médicos recién colegiados de Lima, Perú 2010. Rev Peru Med Exp Salud Publica. 2011;28(2):186-93.

30. Perú, Congreso de la República. Constitución política del Perú. Lima: Congreso de la República; 1993.

31. Tribunal Constitucional. Sentencia del Tribunal Constitucional del 11 de noviembre del 2003, Fundamento jurídico 26.c. Expediente 00082003-AI/TC. Lima: Tribunal Constitucional; 2003.

32. Tribunal Constitucional. Sentencia del Tribunal Constitucional del 23 de mayo del 2014, Fundamentos jurídicos $35 \mathrm{al}$ 59. Expediente 15-2013-PI/TC. Lima: Tribunal Constitucional; 2014.

33. Tribunal Constitucional. Sentencia del Tribunal Constitucional del 26 de marzo del 2003, Fundamento jurídico 3. Expediente 0261-2003-AA/TC. Lima: Tribunal Constitucional; 2003.
34. Tribunal Constitucional. Artículo 100 de la Ley N² 28237 - Código Procesal Constitucional. Lima: Tribunal Constitucional; 2004.

35. Juricic B, Cantuarias R. [Rural health in Chile: A program to atract physicians to rural areas]. Bol Oficina Sanit Panam. 1975 Mar;78(3):203-11. [Artículo en español]

36. Peña S, Ramirez J, Becerra C, Carabantes J, Arteaga O. The Chilean Rural Practitioner Programme: a multidimensional strategy to attract and retain doctors in rural areas. Bull World Health Organ. 2010 May;88(5):371-8. doi: 10.2471/BLT.09.072769.

37. Academia Peruana de Salud. Opinión técnica de la Academia sobre ocho proyectos de ley: Servicio de Medicina y Salud Comunitaria - SERMEC. Rev Acad Peru Salud. 2005;12(2):19-23.

1. Barnighausen T, Bloom DE. Financial incentives to return of service in underserved areas: a systematic review. BMC Health Serv Res. 2009 May 29;9:86. doi: 10.1186/1472-6963-9-86.
2. Miranda JJ, Diez-Canseco F, Lema C, Lescano AG, Lagarde M, Blaauw D, et al. Stated preferences of doctors for choosing a job in rural areas of Peru: a discrete choice experiment. PLoS One. 2012;7(12):e50567. doi: 10.1371/ journal.pone.0050567.

3. Huicho L, Miranda JJ, Diez-Canseco F, Lema C, Lescano AG, Lagarde M, et al. Job preferences of nurses and midwives for taking up a rural job in Peru: a discrete choice experiment. PLoS One. 2012;7(12):e50315. doi: 10.1371/ journal.pone.0050315.

Correspondencia: Percy Mayta Tristán Av. Brasil 2169, Dpto 802, Jesús María, Lima, Perú

Teléfono: (511) 987532133

Correoelectrónico:percy.mayta@upc.edu.pe

\section{Consulte las ediciones anteriores de la} Revista Peruana de Medicina Experimental y Salud Pública en www.scielosp.org

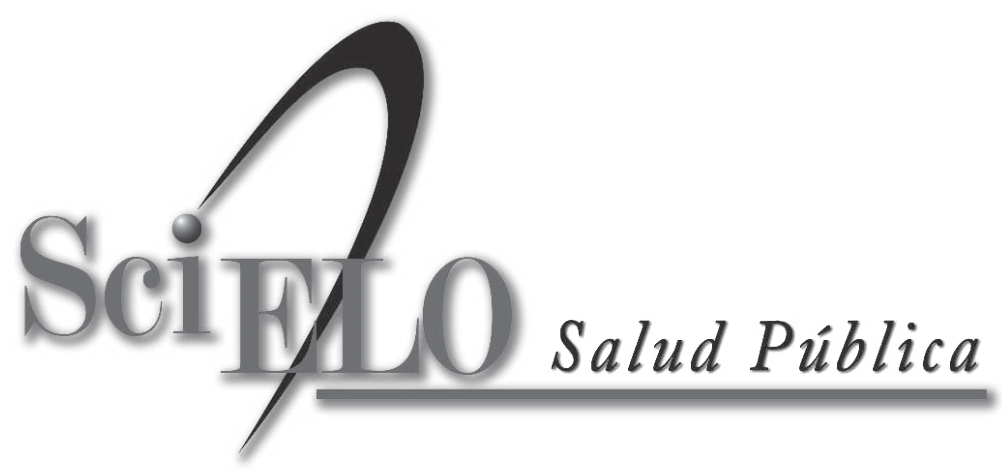

